\title{
Terapia ocupacional e a inclusão digital de pessoas com deficiência*
}

\section{Occupational therapy and digital inclusion of people with disabilities}

\author{
Vanessa da Costa Rosa ${ }^{1}$, Eucenir Fredini Rocha ${ }^{2}$
}

ROSA, V. da C.; ROCHA, E. F. Terapia ocupacional e a inclusão digital de pessoas com deficiência. Rev. Ter. Ocup. Univ. São Paulo, v. 17, n. 3, p. 99-107, set./dez., 2006.

RESUMO: Esse artigo apresenta uma discussão sobre a necessidade da inclusão digital na sociedade atual, e seus benefícios para as pessoas com deficiência. Os terapeutas ocupacionais podem contribuir no sentido de aumentar o nível de participação social de pessoas com deficiência, possibilitando a elas o acesso às tecnologias de informação por meio do uso de diversos recursos, como produtos de tecnologia de apoio. Também acreditamos que o terapeuta ocupacional é o profissional responsável por assessorar outros profissionais - e a população em geral - sobre o uso correto dos dispositivos de tecnologia de apoio e outros aspectos ligados à inclusão digital. Para esse estudo, foram feitas entrevistas com terapeutas ocupacionais de importantes serviços de reabilitação, além de estudos de acessibilidade em serviços de inclusão digital, localizados na Zona Leste da cidade de São Paulo.

DESCRITORES: Terapia ocupacional. Pessoas portadoras de deficiência. Tecnologia da informação. Informática.

\footnotetext{
* Este artigo traz os resultados de Pesquisa de Iniciação Científica financiada pela FAPESP, Processo no. 04/09952-5, período novembro de 2005 a dezembro de 2006.

1 Terapeuta Ocupacional formada pela Universidade de São Paulo. Aprimoranda da Divisão de Medicina de Reabilitação do Hospital das Clínicas da Faculdade de Medicina da Universidade de São Paulo (DMR-HCFMUSP). e-mail: nessa_tousp@yahoo.com.br.

2 Profa. Dra. do Departamento de Fisioterapia, Fonoaudiologia e Terapia Ocupacional da FMUSP, Mestre em Psicologia Escolar/IPUSP e Doutora em Psicologia Social/IPUSP. e-mail: eucenir@usp.br.

Endereço para correspondência: Departamento de Fisioterapia, Fonoaudiologia e Terapia Ocupacional da FMUSP. Rua Cipotânea, 51. CEP: 05360-000. São Paulo, SP.
} 


\section{APRESENTAÇÃO}

$\mathrm{E}$ ste artigo apresenta e analisa os resultados de pesquisa realizada com os objetivos de investigar o estado atual da terapia ocupacional na área de inclusão digital, no município de São Paulo; levantar, no mercado nacional, a existência e as condições de acesso às Tecnologias de Apoio $(\mathrm{TA})^{(1)}$ capazes de tornar a informática acessível a pessoas com diferentes tipos de dificuldades e mapear as condições de inclusão digital em uma região periférica da cidade de São Paulo.

Para tanto, foram realizadas visitas a três instituições de reabilitação: Divisão de Medicina de Reabilitação do Hospital das Clínicas da Faculdade de Medicina da Universidade de São Paulo (DMR-HCFMUSP); Instituto de Ortopedia e Traumatologia do Hospital das Clínicas da Faculdade de Medicina da Universidade de São Paulo (IOTHCFMUSP) e Associação Educacional Quero-Quero. Estas instituições foram escolhidas por serem tradicionais na área de reabilitação na cidade de São Paulo e por afirmarem ter a inclusão social de pessoas com deficiências como objetivo final de seu trabalho.

Em cada uma das instituições citadas, foi entrevistado um terapeuta ocupacional, com o objetivo de detectar, na proposta institucional de reabilitação, o lugar atual da inclusão digital, além de compreender as ações específicas realizadas por terapeutas ocupacionais que atuam nesta área, $\mathrm{O}$ roteiro abordou temas como a caracterização da instituição quanto à sua natureza; seus os objetivos institucionais quanto às atividades de inclusão digital; a caracterização das pessoas atendidas e dos encaminhamentos para inclusão digital; a composição das equipes; os recursos tecnológicos disponíveis e utilizados; os conhecimentos técnicos necessários; a finalidade da inclusão digital junto aos usuários do serviço de reabilitação; a média de custo dos dispositivos utilizados.

Os dados obtidos foram analisados e comparados com a produção literária nacional e internacional que trata da terapia ocupacional e do uso das Tecnologias de Informação e Comunicação (TICs) ${ }^{(2)}$. Foi, ainda, realizado um levantamento dos dispositivos tecnológicos de apoio voltados para a área da informática, disponíveis na Internet e em catálogos de empresas que os comercializam. As principais características dos produtos foram analisadas, bem como o preço e a forma de obtenção, com o objetivo de traçar um panorama sobre a situação do mercado de tecnologia de apoio no Brasil. Ao final, foi elaborado um banco de dados com equipamentos de tecnologia de apoio na área das TICs.

Para mapear as condições de inclusão digital na cidade de São Paulo, foi verificada e analisada a acessibilidade de oito telecentros localizados na região de Itaquera, quais sejam: Parque do Carmo, Luz e Verdade/Vila Carmosina, Dom Bosco, Dom Bosco II, CEU Aricanduva, Jardim Vila Nova, João Batista Conti e Cangaíba. Inicialmente, somente os setes primeiros telecentros citados foram escolhidos para a realização da pesquisa, por estarem localizados em uma área onde o REATA (Laboratório de Reabilitação e Tecnologia Assistiva da USP) promove a inclusão de crianças com deficiência nas escolas municipais. A visita ao telecentro Cangaíba ocorreu por meio de convite da Secretaria Municipal da Pessoa com Deficiência e Mobilidade Reduzida (Seped), como uma contribuição complementar.

\section{A importância da inclusão digital no mundo contemporâneo}

A partir da década de 80, devido a avanços tecnológicos e a políticas de redução dos custos (DUDUCHI, 1998), o uso de computadores pessoais popularizou-se, no Brasil. Assim, em virtude de sua rapidez e praticidade, esta tecnologia passou a ser incorporada ao cotidiano de uma parcela da população que vive, principalmente, nos grandes centros urbanos. O mercado de trabalho, desde então, exige cada vez mais conhecimentos relativos ao uso do computador e da

\footnotetext{
(1) O termo Tecnologia de Apoio foi definido pela Empowering Users Through Assistive Technology (EUSTAT, 1999) e foi escolhido para utilização nesse trabalho por ser considerado mais abrangente que Tecnologia Assistiva. Segundo a EUSTAT, a palavra tecnologia não indica apenas objetos físicos, equipamentos, adaptações de utensílios etc, mas refere-se genericamente a produtos, contextos organizacionais ou modos de agir que formam princípios e componentes técnicos. O termo "apoio" é aplicado à tecnologia quando usada na compensação de uma limitação funcional e na facilitação da vida independente de pessoas com deficiência e/ou idosos. A EUSTAT considera que o profissional ao escolher os dispositivos de tecnologia de apoio, deve levar em conta, além dos fatores individuais, o meio em que o usuário está inserido. O profissional que atua dentro dessa perspectiva, é responsável pela avaliação da eficácia do dispositivo, a viabilidade de seu uso no ambiente do usuário, sua relação com o modo de vida e a personalidade da pessoa.

(2) Segundo o CECAE - USP, TICs são "todas as tecnologias utilizadas na captação, transformação e disseminação de dados, informações e conhecimentos, voltados para as tomadas de decisões pelos dirigentes de organizações publicas e/ou privadas". Nessa pesquisa, os focos são as tecnologias dependentes de redes de dispositivos microprocessados.
} 
Internet, de modo que, hoje em dia, é quase impossível conseguir emprego sem que se tenha, pelo menos, noções básicas de informática.

No entanto, mesmo que, em virtude de políticas de redução de impostos, os preços dos computadores estejam baixando, a verdade é que os benefícios decorrentes do uso da informática ainda não são acessíveis a todas as parcelas da população. Dentre os setores excluídos estão, principalmente, aquelas pessoas com baixo nível sócioeconômico para as quais o custo dos equipamentos disponíveis no mercado brasileiro ainda é proibitivo. Sendo assim, dentre os segmentos populacionais sem acesso à informática, estão pessoas com deficiência que, segundo a Disability Awareness in Action (DAA, 1996), encontramse, em sua maioria, em todas as partes do mundo, entre as pessoas mais pobres dentre os pobres.

Para agravar esta situação, uma das premissas gerais do documento elaborado por Martins et al. (2004), durante a Plenária Final da Oficina para inclusão Digital, que aconteceu no Centro de Convenções Ulysses Guimarães, em Brasília de 14 a 17 de maio de 2001, afirma que "a exclusão digital aprofunda a exclusão sócio-econômica”, pois afasta ainda mais as populações menos preparadas das exigências do mercado de trabalho e do acesso à informação.

No entanto, a exclusão das pessoas com deficiência à informática não se dá apenas por causas financeiras. Mesmo quando é oferecido gratuitamente em espaços públicos, esta parcela da população pode ter limitações motoras, sensoriais e/ou cognitivas que dificultam ou impedem a utilização dos equipamentos. Assim sendo, para que as pessoas com deficiência se beneficiem de todo o potencial positivo das TICs, segundo Martins Neto e Sollemburgo (s. d.), é preciso eliminar barreiras ambientais e sociais; criar e adotar programas adequados a elas; divulgar conteúdos em formatos alternativos e oferecer dispositivos de tecnologia de apoio.

Em virtude de todos esses entraves, segundo o site 1 a 1 (www.1a1ec.com.br) da E-Consulting Corp., apesar de o acesso estar aumentando, apenas $6 \%$ da população brasileira utilizam as TICs. Para aumentar este número, tornase necessário adotar medidas que facilitem a utilização deste tipo de tecnologia, especialmente, com o incentivo à interação entre as empresas, o setor público e o terceiro setor.

As políticas e programas governamentais voltadas à inclusão digital têm como principais objetivos propiciar acesso físico regular aos recursos de tecnologia digital; oferecer oportunidade para que as pessoas adquiram os conhecimentos necessários para utilizar as TICs e acessar a Internet com um mínimo de proficiência.
Para Viana (2001), são vários os motivos pelos quais os governos deveriam investir mais na inclusão digital. Dentre eles, está o crescimento das empresas dedicadas às tecnologias de informação, responsáveis por uma parcela cada vez maior do PIB brasileiro (atualmente, cerca de 10\%). Além disso, sabe-se que a Internet pode ser um meio muito eficiente para a democratizar a informação, com potencial para colocar o governo em linha direta com a população, desde que esta tenha acesso aos equipamentos e domínio dos recursos de tecnologia digital.

Segundo Seabra (s.d), a inclusão digital não se faz por meio de cursos, mas, sim, através da criação de contextos para a utilização do computador. Faz-se necessário identificar as demandas da população e, por meio do trabalho de monitores, permitir que as pessoas tenham acesso às informações de que necessitam e às quais têm interesse, como, por exemplo, serviços de saúde, serviços bancários, receitas de culinária, busca de emprego, informações sobre serviços públicos etc. $\mathrm{O}$ autor também defende a "inclusão dos não excluídos", pois, as ações devem integrar todos os setores da sociedade para tecer a rede de informações.

Os governos dos diversos Estados brasileiros têm reconhecido a importância de políticas públicas para combater a exclusão digital. Atualmente, uma das iniciativas mais difundidas, no País, são os telecentros. O termo telecentro engloba projetos, desenvolvidos em diversas partes do mundo, cujo objetivo é universalizar o acesso às tecnologias digitais e capacitar as pessoas para o uso dos computadores e da Internet, através de oficinas e cursos oferecidos gratuitamente.

Segundo Litto (s.d), o telecentro tem como proposta propiciar a inclusão digital a pessoas de classes econômicas desfavorecidas, de modo a que esta população ganhe maior poder e participação social, à medida que aumenta seu acesso à informação. Assim, os telecentros constituem-se numa ferramenta útil para diminuir a situação de desvantagem de populações que vivem nos grandes centros urbanos.

\section{O acesso as TICs no município de São Paulo}

A cidade de São Paulo conta com três principais tipos de serviços de inclusão digital: os telecentros mantidos pela Prefeitura; os infocentros mantidos pelo governo do Estado e as Escolas de Informática e Cidadania (EICs), criadas pelo Comitê para Democratização da Informática (CDI), em parceria com associações comunitárias, ONGs, órgãos governamentais e instituições que atendem populações vulneráveis.

Segundo dados do site www.prefeitura.sp.gov.br da 
cidade de São Paulo (2005), 105 telecentros foram abertos em locais cujo Índice de Desenvolvimento Humano (IDH) é pior. Há dois tipos de telecentros: os diretos, instalados em prédios ou terrenos da prefeitura, e os conveniados, instalados em espaços cedidos por entidades ou associações conveniadas. Nos dois casos, a manutenção do local e a contratação de profissionais são de responsabilidade do município.

Os infocentros são uma iniciativa do governo do Estado de São Paulo, conduzido pela Imprensa Oficial e pela Companhia de Processamento de Dados do Estado de São Paulo (PRODESP). Existem três tipos de infocentros: os comunitários, criados em parceria com entidades comunitárias, tais como as associações de bairros; os municipais, criados em parceria com as prefeituras; e os Postos Públicos de Acesso à Internet (POPAIs), criados em parceria com órgãos do próprio governo do Estado, em locais de alta circulação, como, por exemplo, os Poupatempos e os terminais de ônibus.

Segundo dados do site Acessa São Paulo (www.acessasaopaulo.sp.gov.br), há na cidade de São Paulo 70 infocentros. Cada unidade possui, em média, 11 computadores modelo Pentium III, ou equivalentes, conectados à Internet banda larga. É permitido que o usuário utilize gratuitamente o serviço por trinta minutos.

As Escolas de Informática e Cidadania (EICs) são espaços informais de ensino, cujo objetivo é atender pessoas em situação de risco social, que vivem em comunidades de baixa renda. Segundo dados do site do CDI São Paulo (www.cdisaopaulo.org.br), existem 40 EICs no município. Através de levantamento de sua localização, sabe-se que grande parte destas escolas funciona em instituições como Febem e penitenciárias, portanto, não são abertas à população em geral.

Gil (2005) lembra que para tornar um telecentro acessível não basta apenas ser solidário com as pessoas que têm deficiência, mas, também, é preciso propiciar o acesso a todas as pessoas, através da adoção do desenho universal. Uma boa acessibilidade favorece não somente pessoas com deficiência, mas também idosos, mulheres grávidas, mães com carrinhos de bebês, pessoas com problemas cardíacos, obesos, pessoas com dificuldades temporárias de locomoção, pessoas carregando objetos pesados etc.

Desse modo, para que os telecentros possam receber pessoas com deficiência é necessário considerar a acessibilidade física ao e no local (guias rebaixadas, rampas, banheiros adaptados etc) e a acessibilidade digital.

Assim, a partir das normas estabelecidas pela Associação Brasileira de Normas Técnicas (ABNT, 2004), foi preparada e utilizada uma lista de conferência com itens referentes à acessibilidade ambiental e às características do mobiliário. As barreiras encontradas foram fotografadas e, a partir desses dados, foram feitas as sugestões para tornar os espaços e os equipamentos plenamente acessíveis.

\section{RESULTADOS}

\section{Terapia ocupacional e inclusão digital}

A partir do levantamento bibliográfico e das entrevistas realizadas, constatou-se que a Terapia Ocupacional não possui, ainda, um conjunto de propostas e estratégias organizadas para o trabalho na área da inclusão digital.

O uso das TICs por terapeutas ocupacionais, em geral, tem se limitado aos espaços das instituições de saúde e voltado a aspectos individuais, com finalidades clínicas, com dificuldades no diálogo com outras áreas, principalmente, no que se refere à implementação dos preceitos das TICs apresentados no início deste trabalho.

Pela perspectiva dos terapeutas ocupacionais entrevistados, a inserção da pessoa com deficiência no espaço social ocorre a partir de seu esforço próprio e, para tanto, os recursos da inclusão digital estão circunscritos ao processo terapêutico de cada usuário e disponíveis no ambiente do serviço de reabilitação.

As pessoas são preparadas dentro da instituição para utilizarem as TICs e, após uma espécie de treinamento, considera-se que estão aptas para utilizar esses recursos no contexto familiar e social.

Outro aspecto do trabalho da Terapia Ocupacional, nas instituições visitadas, é o fato de que as TICs são utilizadas como recursos terapêuticos durante o processo de reabilitação, na medida em que ajudam a desenvolver capacidades como raciocínio, coordenação motora, orientação temporal-espacial, agilidade etc.

Os terapeutas ocupacionais entrevistados não manifestaram preocupação em atuar diretamente para garantir acessibilidade aos equipamentos de TICs. Esta responsabilidade é repassada aos clientes, desconsiderando-se que as inúmeras dificuldades existentes, em nosso meio social, tornam esta uma missão quase impossível para a maioria das pessoas com deficiência ou suas famílias.

A utilização das tecnologias de apoio, no processo de inclusão digital, é importante, mas não pode limitar-se somente à indicação e confecção dos dispositivos por terapeutas ocupacionais. Cabe a este profissional também informar e capacitar os monitores de telecentros e professores da rede escolar (PELOSI, 2004), a fim de 
conscientizá-los a respeito dos aspectos específicos relacionados às dificuldades e potencialidade das pessoas com deficiência, bem como sobre a explicitação das possibilidades proporcionadas pelas TAs. Da mesma forma, o terapeuta ocupacional deve diversificar e ampliar seus conhecimentos para que seu trabalho esteja em consonância com os preceitos da inclusão digital. Isto pode ser feito a partir de cursos de pós-graduação latu-sensu, de atualizações teóricas e práticas, bem como através do acesso aos novos equipamentos e programas de TICs.

\section{Produtos de tecnologia de apoio voltados para a inclusão digital de pessoas com diferentes incapacidades disponíveis no mercado nacional}

De acordo com nossa pesquisa, os produtos encontrados dividem-se nas seguintes categorias: produtos nacionais (24\%); softwares gratuitos (17\%); produtos internacionais sem representação no Brasil (20\%) e produtos internacionais com representação nacional (39\%).

Os produtos pesquisados apresentam uma contradição: ao mesmo tempo em que há produtos extremamente caros, como os softwares e periféricos internacionais e, portanto, inacessíveis para a grande maioria da população atendida pelos serviços de saúde e de reabilitação e, conseqüentemente, pela terapia ocupacional no Brasil, também foi possível encontrar softwares de excelente qualidade distribuídos gratuitamente.

Desse modo, um dos grandes problemas parece ser a falta de divulgação, bem como, de acesso ao computador e de capacitação dos profissionais (terapeutas ocupacionais, professores) que poderiam facilitar o uso desses produtos pelas pessoas com deficiências. Seria ideal que existissem outros meios de divulgação e que esses softwares estivessem disponíveis em locais públicos como os telecentros.

Através da identificação das necessidades das pessoas com deficiência e do levantamento de todos os recursos existentes no território nacional, o Ministério da Ciência e Tecnologia (2005), em última análise o responsável por formular políticas públicas para o setor, afirma que o uso de tecnologias de apoio ainda é restrito porque: 1. pessoas com deficiência não têm conhecimento sobre as tecnologias disponíveis; 2. profissionais de reabilitação não estão suficientemente preparados para orientar sua clientela; 3 . não há investimento em pesquisas; 4 . há poucos produtos no mercado nacional e, principalmente, não há incentivo para o desenvolvimento desse tipo de tecnologia.

Como, no Brasil, a fabricação e comercialização das tecnologias de apoio estão, geralmente, ligadas a centros de reabilitação, tais como a AACD, IOT (Instituto de Ortopedia e Traumatologia da FMUSP), Laramara (Laratec), o acesso, treinamento e uso desses produtos ficam restritos a sua clientela. Esses centros de reabilitação, além de produzirem alguns produtos, acabam utilizando grandes marcas disponíveis no mercado. Como o mercado é escasso e não há competitividade, os preços tornam-se inacessíveis para grande parte da população.

Dentre os produtos comercializados no País, cerca de $11 \%$ são importados e de custo muito alto. Sendo assim, seu uso fica restrito às classes mais privilegiadas e sua comercialização contribui muito pouco para a inclusão digital, visto que, segundo dados do IBGE, das vinte e cinco milhões de pessoas com deficiência, no Brasil, cerca de três milhões vivem com menos de um salário mínimo (BRASIL, 2003).

Instituições como DMR e Quero-Quero também produzem, por exemplo, acionadores de teclado, cujo uso é limitado à própria clientela e cuja divulgação restringe-se a apresentações em feiras e congressos. Tais produtos, em geral de baixo custo, poderiam beneficiar muitos usuários, se houvesse maior distribuição e melhor divulgação. Para isso, seria importante aumentar as trocas científicas entre instituições, universidades, empresas de informática e serviços de inclusão digital, através de congressos, por exemplo. No entanto, ao contrário, nota-se uma divulgação restrita dos trabalhos relacionados aos equipamentos, em prejuízo da população que necessita dos produtos de tecnologia de apoio.

Seria importante, também, que empresas e instituições criassem estratégias de divulgação e venda de equipamentos usados e/ou de baixo custo, o que poderia ser feito em feiras, congressos ou sites destinados a pessoas com deficiência como a Rede Saci (www.saci.org.br) e a Rede Entre Amigos (www.entreamigos.com.br).

\section{Condições de acessibilidade nos telecentros do município de São Paulo}

A distribuição dos telecentros na cidade de São Paulo está configurada da seguinte forma: $41 \%$ na Zona Leste; $32 \%$ na Zona Sul; $19 \%$ na Zona Norte e $8 \%$ na Zona Oeste. Como objeto de investigação deste trabalho foram avaliados oito telecentros do município de São Paulo, ou seja, cerca de 7\% do total da cidade, todos localizados na região Leste.

Os aspectos avaliados nos telecentros visitados foram: transporte e condições de acesso ao redor e no local, isto é, calçada, entrada, estacionamento, sanitários, mobiliário, hardware e software. Abaixo, são apresentados os dados 
colhidos nos telecentros:

- Transportes

Tivemos grandes dificuldades para obter informações sobre ônibus adaptados que servem aos telecentros visitados. Algumas informações foram obtidas no Conselho Municipal da Pessoa Deficiente (CMPD), porém, a lista de linhas com ônibus adaptados disponível, nesse local, está incompleta.

Segundo dados obtidos na São Paulo Transporte S.A. (SPTrans), empresa responsável pela gestão do sistema de transporte, a frota de São Paulo deveria ter, pelo menos, um veículo adaptado operando em cada linha do município. Porém, esta meta ainda não foi atingida. Como proposta para sanar esta falta, a SPTrans sugere que o próprio usuário contate a empresa de ônibus que serve sua região e solicite que o serviço seja alocado segundo suas necessidades. É evidente que, na prática, esta proposta, além de não funcionar, nega a responsabilidade que a prefeitura tem de oferecer transporte acessível e ainda culpabiliza a pessoa com deficiência pela não existência de um serviço ao qual ela tem direito. Como consequiência desta política equivocada, não existem ônibus adaptados suficientes, o que reduz significativamente as possibilidades de circulação das pessoas com deficiência e, como conseqüência, dificulta, quando não impede, sua participação social e seu acesso às TICs.

- Acesso ao redor do telecentro

Nota-se, em geral, que não há preocupação em proporcionar acesso conforme as normas estabelecidas pela ABNT. Durante as visitas, encontramos diversos obstáculos, tais como pisos irregulares, buracos e mato alto nas calçadas, além de degraus, ausência de guias rebaixadas e de rampas de acesso em $100 \%$ dos locais.

\section{. Estacionamento}

Dentre os oitos telecentros visitados, somente três deles, localizados dentro de entidades, possuem estacionamento que, no entanto, não são de uso exclusivo dos usuários dos telecentros. Nenhum deles tem vagas reservadas para veículos de pessoas com deficiência. São eles: Telecentro Parque do Carmo (localizado no interior do Centro Educacional Esportivo Rumi de Ranieri), Telecentro CEU Aricanduva e Telecentro Dom Bosco.

.Entrada

Em 56\% dos telecentros avaliados, foram encontrados obstáculos na entrada. Somente $22 \%$ deles possuem rampa e os outros $22 \%$ têm entradas ao nível da rua. Mesmo os que têm alguma acessibilidade apresentam dificuldades, como, por exemplo, um degrau no final de uma rampa que, por vezes, está quebrada.

\section{- Sanitários}

Em 100\% dos telecentros pesquisados, mesmo naqueles nos quais há algum tipo de adaptação, como a elevação da altura do vaso sanitário, as condições de acessibilidade são precárias. Em um deles, por exemplo, o sanitário é utilizado como depósito. Em outros, há dificuldade de circulação de cadeira de rodas, pois as portas são estreitas ou não estão posicionadas de acordo com o padrão da ABNT. Outra dificuldade encontrada é o trajeto de difícil circulação até os sanitários. São uma constante os corredores estreitos e obstruídos por materiais diversos.

\section{. Mobiliário}

Quanto ao mobiliário, verifica-se que os telecentros obedecem a um padrão que, em sua maioria, permite o uso por pessoas com deficiência. Somente em um dos telecentros pudemos verificar que a largura e altura da mesa não estavam adequadas. O problema maior está na disposição do mobiliário que impede ou atrapalha a circulação dentro do telecentro.

\section{- Acessibilidade digital}

Quanto à acessibilidade digital, todos os telecentros são iguais. Utilizam o sistema operacional GNU/Linux, que contém opções de acessibilidade semelhantes ao Windows (sistema operacional mais utilizado no País). Nenhum telecentro conta com dispositivos de tecnologia de apoio, como, por exemplo, software para cegos.

Portanto, pode-se afirmar que, no que diz respeito à acessibilidade, as condições de acessibilidade dos telecentros pesquisados deixam muito a desejar. Apenas alguns apresentam adaptações, mas que estão em desacordo com as normas propostas pela ABNT. Nota-se, portanto, falta de informação a respeito das necessidades dos usuários com deficiência, o que torna o acesso difícil, ou até mesmo impossível.

Em todos os telecentros visitados, os monitores mostravam-se surpresos com o foco da nossa pesquisa, já que, pela experiência deles, havia pouca participação de pessoas com deficiência no telecentro. Muitos questionaram o propósito do nosso trabalho: Para que adaptar um telecentro se não há pessoas com deficiência utilizando esse serviço?

O papel dos governos é formular políticas públicas referentes à prestação de serviços que, de acordo com a Empowering Users Through Assistive Technology 
(EUSTAT), são definidos como:

"conjunto de facilidades, procedimentos e processos que atuam como intermediários entre estruturas de mercado de tecnologia de apoio e os utilizadores finais, de modo a facilitar o acesso das pessoas com deficiência a estas tecnologias, através de ajuda financeira, competência profissional, informação, formação etc.”.

E esses serviços devem ter como pressupostos: acessibilidade, competência, eficiência, flexibilidade (serem capazes de atender a necessidades individuais) e levarem em conta a opinião das pessoas com deficiência.

O documento elaborado em 2001, durante a Oficina para a Inclusão Digital, afirma que a inclusão digital para as pessoas com deficiência é mais importante do que para as demais, em virtude do potencial para gerar oportunidades e desdobramentos positivos que tal inserção possui. Porém, é exatamente esta parcela da população que não é devidamente considerada quando se elaboram políticas públicas de inclusão digital.

Desse modo, a questão é outra: Como é possível que pessoas com deficiência utilizem os telecentros, sendo que eles não são acessiveis? Ao construir um local, sem levar em consideração as normas de acessibilidade, a exclusão da população com deficiência está dada a priori.

Se serviços para uso exclusivo de pessoas com deficiência reproduzem as dinâmicas institucionais, reforçam a segregação e a exclusão e, portanto, são contrários aos preceitos de uma sociedade inclusiva e da própria política de incentivo à inclusão digital, ao constatar que não existe uma política séria para oferecer acessibilidade a esse segmento da população nos telecentros, podemos concluir que as pessoas com deficiência não são percebidas como parte da sociedade e, muito menos, como um público que também precisa, pode e quer beneficiar-se da informática.

\section{CONCLUSÃO}

Consideramos o terapeuta ocupacional como uma peça chave na capacitação de pessoas com deficiência para utilizarem as TICs, pois é o profissional habilitado para oferecer apoio aos usuários dos serviços de reabilitação, às escolas e aos telecentros, no que tange à introdução de equipamentos e programas adequados e à orientação sobre como tornar acessíveis os espaços físicos e o mobiliário.

Entretanto, através da coleta de dados obtidas na literatura e pelas entrevistas feitas para esta pesquisa, concluímos que o terapeuta ocupacional tem atuado de forma limitada e tímida na área da inclusão digital, ao restringir seu trabalho à ação nas instituições de reabilitação e não aprofundar o diálogo com profissionais de fora do âmbito da saúde.

Para a EUSTAT, as etapas de formação dos utilizadores de tecnologias de apoio são:

- Campanhas de sensibilização: voltadas à modificação das atitudes gerais do público;

- Informação: difusão das novidades para uma vasta audiência de pessoas interessadas;

. Ensino e treino: aumento dos conhecimentos e competência das pessoas em determinado tópico, o público geralmente é restrito;

- Aconselhamento: chave para a solução de problemas individuais específicos.

No entanto, esta pesquisa constatou que o trabalho dos terapeutas ocupacionais, geralmente, está ligado apenas à última etapa referida, isto é, de aconselhamento, pois faz a avaliação das características físicas e cognitivas, prescreve e treina diretamente o usuário das tecnologias de apoio que dão acesso às TICs. Todavia, o trabalho do terapeuta ocupacional pode ser ampliado para as outras etapas citadas pela EUSTAT, pois ele está capacitado para repassar as informações aos outros profissionais, por meio de cursos, workshops, conferências etc.

Embora seja de extrema importância que os terapeutas ocupacionais dominem melhor o assunto, caso queiram ter uma atuação mais eficaz na área, durante o desenvolvimento desta pesquisa, notamos que os entrevistados não se preocupam em se apropriar dos conhecimentos produzidos pela área da inclusão digital. Portanto, é importante que o terapeuta ocupacional esteja disposto também a aprender com profissionais de outras áreas, sobretudo, aqueles ligados à inclusão digital.

O terapeuta ocupacional também pode, por meio do diálogo com os produtores de equipamentos, tornar mais acessíveis os produtos de tecnologia de apoio. Da mesma forma, os terapeutas ocupacionais podem buscar formas de facilitar a disponibilidade, a redução dos custos e, até mesmo, garantir a gratuidade dos produtos, visto que grande parte da população com deficiência tem baixa renda.

É possível, ainda, aumentar o acesso aos produtos e incentivar o empoderamento de sua clientela, ao oferecer condições para que as pessoas com deficiência e suas famílias utilizem a Internet e os recursos informativos existentes - tais como bases de dados, catálogos e exposições comerciais - , para buscar, por si mesmas, as informações de que necessitam. Além disso, é necessário que os próprios terapeutas ocupacionais produzam informações sobre o assunto, promovam eventos e 
publiquem seus trabalhos.

Um outro meio simples e eficaz de difundir conhecimentos é promover encontros entre utilizadores de tecnologia de apoio e capacitar as próprias pessoas com deficiência para difundirem informações e trocarem experiências sobre o assunto, tanto entre seus pares quanto entre os profissionais.

As medidas aqui propostas visam à eliminação dos fatores - frutos do "esquecimento" dos governos e dos profissionais - que constituem barreiras à inclusão digital das pessoas com deficiência. Para tanto, é necessária uma atuação interdisciplinar, na qual o terapeuta ocupacional tem grande relevância, pois possui conhecimentos importantes sobre a deficiência, o uso das tecnologias de apoio e os conceitos de acessibilidade e inclusão social que devem ser considerados na formulação de estratégias para a inclusão digital.

Ao ajudar a eliminar as barreiras psicossociais e oferecer assessoria para melhorar as condições de acessibilidade física e digital, o terapeuta ocupacional pode ter uma atuação fundamental para a difusão e troca de conhecimentos e, mais importante, para o exercício da cidadania nos telecentros, tornando-os espaços de fato inclusivos.

E nossa proposta, cumpre destacar, é para que o acesso a todos os telecentros se baseie nos princípios do Desenho Universal, ou seja, sejam acessíveis a todas as pessoas, incluindo aquelas que têm deficiências. Somente desta forma, os telecentros se tornarão realmente espaços que visam à inclusão social de todos.

ROSA, V. da C.; ROCHA, E. F. Occupational therapy and digital inclusion of people with disabilities.

Rev. Ter. Ocup. Univ. São Paulo, v. 17, n. 3, p. 99-107, set./dez., 2006.

\begin{abstract}
This article presents a discussion about the necessity of digital inclusion in actual society and its benefits for disabled people. Occupational Therapists can contribute in order to increase social participation level of disabled people, giving them access to information technology through the use of several resources, such as assistive technology products. We also believe that Occupational Therapist is the professional responsible for advise other professionals and general population about the correct use of assistive technology products, and other aspects connected to the digital inclusion. For this study, interviews were realized with occupational therapists from important rehabilitation services in São Paulo and also acessibility studies in digital inclusion services localizated in East Area of São Paulo city.
\end{abstract}

KEY WORDS: Occupational therapy. Informatics. Information technology. Disabled persons.

\title{
REFERÊNCIAS
}

\begin{abstract}
ASSOCIAÇÃO BRASILEIRA DE NORMAS TÉCNICAS (ABNT). Acessibilidade a edificações, mobiliário, espaços $e$ equipamentos urbanos. Rio de Janeiro, 2004.
\end{abstract}

ASSOCIAÇÃO BRASILEIRA DE NORMAS TÉCNICAS. Acessibilidade de pessoas portadoras de deficiências a edificações, espaço, mobiliário e equipamentos urbanos. Rio de Janeiro, 1994.

BRASIL. Casa Civil. Programa "Braços Abertos": inclusão social para portadores de deficiência, 2003. Disponível em: http:// presidencia.gov.br/casacivil/site/exec/noticias.cfm?cod=29. Acesso em 06/07/2004.

DAA - DISABILITY AWARENESS IN ACTION. Idéias práticas em apoio ao Dia Internacional das Pessoas com Deficiência: 3 de dezembro, um dia para promover os direitos humanos de todas as pessoas portadoras de deficiência. São Paulo:

\section{PRODEF/APADE, 1996.}

DUDUCHI, M. A internet e seus benefícios a pessoas portadoras de deficiências. In: Tecnologia em (Re)habilitação cognitiva uma perspectiva multidisciplinar. São Paulo: EDUNISC, 1998.

EUSTAT. Educação em tecnologias de apoio para utilizadores finais: linhas de orientação para formadores. Comissão Européia: 1999. Disponível em: http://www.siva.it/research/eustat/ estsgupt.html. Acesso: 02 maio. 2004.

MARTINS, A.; AFONSO, C. A.; ASSUMPÇÃO, R.; BARCELLOS, S. Oficina para a inclusão digital. Disponível em: http://www.cidec.futuro.usp.br/. Acesso em: 02 maio 2004.

MARTINS NETO, J. C.; SOLLEMBERGO, R. S. Tecnologias assistivas e a promoção da inclusão social. Disponível em: 
ROSA, V. da C.; ROCHA, E. F. Terapia ocupacional e a inclusão digital. Rev. Ter. Ocup. Univ. São Paulo, v. 17, n. 3, p. 99-107, set./dez., 2006.

http://www.saci.org.br/index.php?modulo=arkemi\& parametro=15515; http://www.saci.org.br/index.php?modulo= akemi\&parametro $=15515$. Acesso em 30 mar. 2005 .

PELOSI, M. B. A comunicação alternativa e ampliada nas escolas do município do Rio de Janeiro. In: NUNES, L. R. O. P. (Org.). Favorecendo o desenvolvimento da comunicação em crianças e jovens com necessidades educacionais especiais. Rio de Janeiro: Dunya, 2004. p. 63-75.

PELOSI, M. B. A comunicação alternativa escrita. In: NUNES, L. R. O. P (Org.). Favorecendo o desenvolvimento da comunicação em crianças e jovens com necessidades educacionais especiais. Rio de Janeiro: Dunya, 2004. p. 205-216.

ROCHA, E. F. Recursos tecnológicos e equipamentos. In: SIMPÓSIO LATINO-AMERICANO DE TERAPIA OCUPACIONAL, 4; CONGRESSO BRASILEIRO DE TERAPIA OCUPACIONAL, 5. Anais... Belo Horizonte, 1997. p.161-9.

ROCHA, E. F.; CASTIGLIONI. M. C. Reflexões sobre recursos tecnológicos: ajudas técnicas, tecnologia assistiva, tecnologia de assistência e tecnologia de apoio. Rev. Ter. Ocup. Univ. São Paulo, v. 16, n. 3 p. $97-104,2005$.

Recebido para publicação: Dez/2006

Aceito para publicação: Dez./2006 